\title{
AVALIAÇÃO DOS TEORES DE ZINCO EM BRÂNQUIAS, CARCAÇA, FÍGADO E MUSCULATURA DE DIFERENTES ESPÉCIES DE PEIXES CAPTURADOS NO RIO SÃO FRANCISCO (MG, BRASIL)
}

\author{
Carla Rosa Labarrère ${ }^{1}$; Bruna Dias Menezes ${ }^{1}$, Marília Martins Melo ${ }^{1 *}$ \\ 1 - Escola de Veterinária, Laboratório de Toxicologia, Universidade Federal de Minas Gerais, Caixa-Postal: 567, Avenida Antônio Carlos, 6627, \\ Campus universitário , 31270-901 - Belo Horizonte, MG - Brasil, * autor para correspondência: mariliamm@ufmg.br
}

Recebido em 22 de abril de 2012; aceito em 23 de maio de 2012

\begin{abstract}
RESUMO: O zinco (Zn) é considerado indispensável para o funcionamento de muitas atividades celulares nos peixes. Entretanto, quando em excesso, pode tornar-se nocivo ao organismo. A bioacumulação de $\mathrm{Zn}$ nos peixes é uma ferramenta importante para avaliação da poluição ambiental. Diante deste contexto, objetivou-se dosar os teores de Zn nas brânquias, carcaça, fígado e musculatura de 68 peixes (totalizando 272 amostras) das espécies Hoplias sp., Hypostomus sp., Piaractus sp., Pimelodus sp. e Prochilodus sp., capturados em cinco distintos pontos do Rio São Francisco (Minas Gerais) no ano de 2008. A área de estudo compreendeu a região de Três Marias, desde o Reservatório da Usina Hidrelétrica da CEMIG até a foz do rio Abaeté. Vinte e oito animais apresentaram concentrações de $\mathrm{Zn}$ acima do permitido pela legislação brasileira $(50,00 \mathrm{mg} / \mathrm{kg})$ em pelo menos uma de suas vísceras. Do total das 272 amostras avaliadas, $50(18,38 \%)$ estavam inadequadas. As espécies Hoplias sp. e Hypostomus sp. apresentaram a maior bioacumulação de $\mathrm{Zn}$ no fígado e na carcaça. $\mathrm{O}$ valor médio de $\mathrm{Zn}$ na musculatura foi de $6,82 \pm 3,00 \mathrm{mg} / \mathrm{kg}$, ou seja, todas amostras de musculatura apresentaram baixos valores deste metal. Não houve diferença na concentração do $\mathrm{Zn}$ entre os cinco pontos estudados. Conclui-se que o tecido hepático da espécie de peixe Hoplias sp., é o melhor biomarcador da contaminação de Zn.
\end{abstract}

Palavras-chave: Zinco, peixe, Rio São Francisco.

ABSTRACT: ZINC EVALUATION IN GILLS, CARCASS, LIVER AND MUSCLE OF DIFFERENT FISH SPECIES CAUGHT IN SÃO FRANCISCO RIVER (MG, BRAZIL). The Zinc (Zn) is considered indispensable for the functioning of many cellular activities in fish. However, in high levels, zinc may become harmful. The bioaccumulation of $\mathrm{Zn}$ in fish is an important tool for assessing environmental pollution. In this context, the aim of the study was to dose the levels of $Z n$ in gills, carcass, liver and muscle of 68 fish (total 272 samples) of: Hoplias sp., Hypostomus sp., Piaractus sp., Pimelodus sp. and Prochilodus sp., caught in five different parts of the São Francisco River (Minas Gerais) in 2008. The studied area covered the region of Três Marias, between CEMIG hydroelectric power plant reservoir and Abaeté River. Twentyeight animals showed $\mathrm{Zn}$ concentrations above the limit level allowed by Brazilian legislation $(50 \mathrm{mg} / \mathrm{kg})$ in at least one of its tissues. Out of 272 samples tested, 50 (18.38\%) were inadequate. The fish Hoplias sp. and Hypostomus sp. presented the highest bioaccumulation of $\mathrm{Zn}$ in liver and carcass. The mean value of $\mathrm{Zn}$ in the muscle was $6.82 \pm 3.00 \mathrm{mg} / \mathrm{kg}$, which means, all muscle samples showed low levels of this metal. There was no difference in $\mathrm{Zn}$ concentration among the five locations studied. It was concluded that the liver of the fish specie Hoplias sp. is the best biomarker of $\mathrm{Zn}$ contamination.

Keywords: Zinc, fish, São Francisco River.

\section{INTRODUÇÃO}

O aumento da população mundial nas últimas décadas está associado à expansão de atividades industriais. A produção de resíduos urbanos e rurais tornou-se então fonte poluidora importante, permitindo a contaminação de recursos hídricos tão valiosos nos dias de hoje (Szyczewski et al. 2009; Ozden, 2010). Dentre os agentes contaminantes, salientam-se os metais pesados devido às consequências decorrentes de sua deposição em ecossistemas aquáticos (Sloman 2007; Marques et al. 2009). Estes ecossistemas são especialmente sensíveis à presença destes agentes tóxicos, devido ao processo de bioacumulação Mendil et al., (2010a).

Uma vez presentes nos cursos hídricos, os metais pesados não só são absorvidos pelos peixes pelo trato gastrintestinal como também pelo epitélio branquial (Kosanovic et al., 2007). Os peixes ficam vulneráveis à contaminação do seu habitat, servindo, portanto, como biomarcadores de poluição (Lins et al., 2010). Alguns metais são considerados nutricionalmente essenciais, como o $\mathrm{Zn}$, por desempenharem funções importantes nos processos biológicos. Estes podem tornar-se nocivos ao organismo quando ingeridos em quantidades muito acima das desejáveis (Amundesen et al., 1997; Ashraf et al., 2006).

Uma vez acumulado em grandes quantidades no organismo dos peixes, o $\mathrm{Zn}$ é capaz de causar alterações histopatológicas nas brânquias como hiperplasia, fusão lamelar, destruição do epitélio e produção excessiva de muco (Hogstrand et al., 1994; Marques, et al., 2009), além de distúrbios no equilíbrio ácido-básico (Hogstrand et al., 1994) e efeitos imunotóxicos (Mottin et al., 2010). Kuz'mina (2011) avaliou o efeito do $\mathrm{Zn}$ sobre o comportamento de peixes (larvas de Chironomus sp.) e observou que a exposição a este elemento é capaz de causar diminuição do consumo de alimentos.

O Rio São Francisco percorre uma extensão de aproximadamente $2.900 \mathrm{~km}$, atravessando os estados de Minas Gerais, Bahia, Pernambuco, 
Alagoas e Sergipe. Sua bacia hidrográfica cobre uma área de $636.920 \mathrm{~km}^{2} \mathrm{e}$, inferindo importância econômica, social e ambiental no território brasileiro. Devido a sua localização geográfica, a bacia do Rio São Francisco está sujeita à contaminação por diversos poluentes provenientes de grandes cidades, polos industriais, mineradoras e atividades agrícolas (Gomes et al., 2010).

Cabe ressaltar que o Rio São Francisco possui um grande número de espécies de peixes com elevado valor econômico tais como Hoplias sp., Piaractus sp., Pimelodus sp., Prochilodus sp. e Hypostomus sp. Estas espécies são importantes devido à relevância nutricional do consumo de peixes na dieta alimentar humana, tanto para a população local como nacional, e por desempenharem papel fundamental na pesca esportiva (Godinho \& Godinho, 2003). Diante do exposto, este trabalho teve como objetivo dosar as concentrações de Zn nas brânquias, fígado, carcaça e musculatura de diferentes espécies de peixes capturados no rio São Francisco (MG).

\section{MATERIAL E MÉTODOS}

A área de estudo foi o Rio São Francisco em Minas Gerais, ao longo de $42 \mathrm{~km}$ de sua extensão (Figura 1). A coleta foi realizada próximo à cidade de Três Marias (MG) em cinco distintos pontos, desde o Reservatório da Usina Hidrelétrica (UHE) de Três Marias até a foz do rio Abaeté (Pontal), cujas coordenadas geográficas são: (1) altitude: $548,78 \mathrm{~m}$ $18^{\circ} 12^{`} 12^{\prime \prime} \mathrm{S}-45^{\circ} 19^{\prime} 28^{\prime \prime} \mathrm{W}$; (2) altitude: $523,78 \mathrm{~m}-$ $18^{\circ} 08^{\prime} 46^{\prime \prime} \mathrm{S}-45^{\circ} 15^{\prime} 53^{\prime \prime} \mathrm{W}$; (3) altitude: $511,28 \mathrm{~m}-$ $18^{\circ} 11^{`} 53^{\prime \prime} \mathrm{S}-45^{\circ} 15^{\prime} 06^{\prime \prime} \mathrm{W}$; (4) altitude: $516,46 \mathrm{~m}-$ $18^{\circ} 08^{\prime} 46^{\prime \prime} \mathrm{S}-45^{\circ} 13^{\prime} 39^{\prime \prime} \mathrm{W}$; (5) altitude: $506,10 \mathrm{~m}-$ $18^{\circ} 02^{\prime} 26^{\prime \prime} \mathrm{S}-45^{\circ} 11^{\prime} 15^{\prime \prime} \mathrm{W}$.

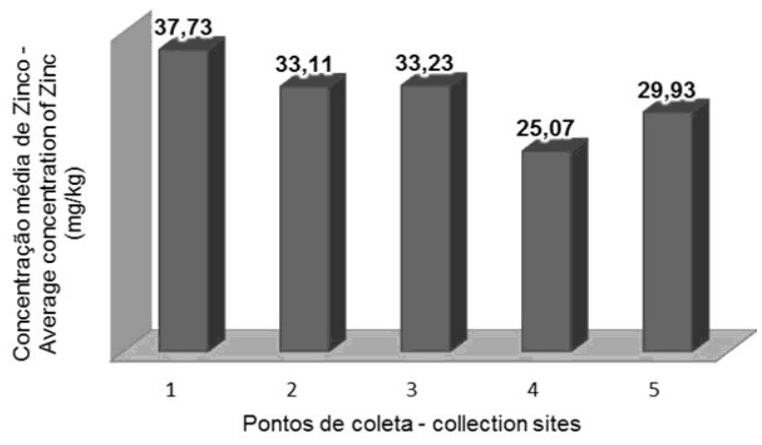

Figura 1 - Concentração média de Zinco (mg/kg; 2008) nos peixes coletados nos pontos (1) altitude: $548,78 m-18^{\circ} 12^{\prime} 12^{\prime \prime} \mathrm{S}$ $45^{\circ} 19^{\prime} 28^{\prime \prime} W$; (2) altitude: $523,78 m-18^{\circ} 08^{\prime} 46^{\prime \prime} S-45^{\circ} 15^{\prime} 53^{\prime \prime} W$; (3) altitude: $511,28 m-18^{\circ} 11^{\prime} 53^{\prime \prime} \mathrm{S}-45^{\circ} 15^{\circ} 06^{\prime \prime} W$; (4) altitude: $516,46 m-18^{\circ} 08^{\prime} 46^{\prime \prime} S-45^{\circ} 13^{`} 39^{\prime \prime} W$; (5) altitude: 506,10m$18^{\circ} 022^{\prime} 6^{\prime} \mathrm{S}-45^{\circ} 11^{`} 15^{\prime \prime} W$; do Rio São Francisco (MG).

Durante todo o ano de 2008, um total de 68 peixes foram capturados, sendo 14 do gênero Hoplias sp., cinco de Hypostomus sp., quatro de
Piaractus sp., 25 de Pimelodus sp. e 20 de Prochilodus sp. (Figura 2). De cada animal, foram coletadas amostras de brânquias, fígado, carcaça e musculatura para análise das concentrações de Zn por espectrofotometria de absorção atômica. A digestão das amostras seguiu o método descrito pela American Public Health Association (1992).

O delineamento aplicado foi inteiramente ao acaso em esquema de Parcelas Subdivididas (Sampaio, 2007). Primeiramente, os pontos de coleta não foram considerados para a análise. Dessa forma, as parcelas corresponderam às espécies e as subparcelas às vísceras. Posteriormente, nova análise foi realizada. Dessa vez, as espécies foram desconsideradas e, portanto, as parcelas passaram a ser os pontos de coleta e as subparcelas as vísceras. Para testar a normalidade e homocedasticidade foram utilizados os teste de Lilliefors e Bartlett, respectivamente. As variáveis apresentaram distribuição normal após sofrerem transformação logarítmica ( $\log 10)$ e, em seguida, foram submetidas à Análise de Variância (ANOVA) e comparação de médias pelo teste de Tukey. A influência do comprimento e peso dos animais sobre a concentração de $\mathrm{Zn}$ foi analisada através da Correlação de Person. Os resultados foram analisados pelo programa SAEG (Sistema para Análises Estatísticas e Genéticas).

\section{RESULTADOS E DISCUSSÃO}

Dos 68 peixes capturados, 28 apresentaram concentração de $\mathrm{Zn}$ superior a $50 \mathrm{mg} / \mathrm{kg}$ (concentração máxima permitida pela legislação brasileira), em pelo menos uma de suas vísceras (ANVISA, 1965). Do total de 272 amostras analisadas (brânquias, carcaça, fígado e musculatura dos 68 animais), 50 (18,38\%) estavam acima do limite permitido. Das amostras impróprias, encontram-se exemplares de fígado, carcaça e brânquias. Contudo, as maiores concentrações de $\mathrm{Zn}$ foram observadas no fígado, o qual teve valores deste metal acima de $100 \mathrm{mg} / \mathrm{kg}$ em cinco amostras, ou seja, o dobro do estipulado pela Anvisa (Tabela 1).

O fígado é o principal local de detoxicação e armazenagem de metais pesados. Isso ocorre devido à capacidade deste órgão em induzir a ligação de metais a proteínas como metalotioneína (Nakayama et al., 2010), além da sua alta atividade metabólica (Agah et al., 2009).

Elevados valores de $\mathrm{Zn}$ na carcaça demonstram que o $\mathrm{Zn}$ pode estar se acumulando gradativamente nos animais, provavelmente decorrente de altos valores presentes na água do Rio São Francisco. Kosanovic et al. (2007) também encontraram valores de $\mathrm{Zn}$ acima de $50 \mathrm{mg} / \mathrm{kg}$ na espécie Lethrinus lentjan da costa ocidental dos Emirados Árabes 
Unidos, assim como Mendil et al. (2010b) que relataram altos valores de $\mathrm{Zn}$ em diferentes espécies de peixes de uma região afetada por dejetos agrícolas e industriais na Turquia.

Há uma crescente preocupação em relação ao risco que os metais acumulados nos peixes representam para a saúde do homem, especialmente para populações que possuem elevada taxa de consumo desta carne segundo Mansilla-Rivera \& Rodríguez-Sierra (2011). Fatores bióticos e abióticos, como as propriedades químicas da água e características específicas das espécies, podem afetar a bioacumulação de metais em organismos aquáticos (Chiu \& Mok, 2010). Peixes são especialmente susceptíveis à contaminação por metais pesados. No organismo, esses elementos tendem a permanecer nos tecidos podendo acumular-se após consecutivas exposições. Em geral, os contaminantes químicos têm maior afinidade por tecidos com alto teor de gordura, mas a concentração diferenciada de um tóxico também é influenciada pela forma como é metabolizado (Palaniappan et al., 2010).

Os resultados mostraram que acúmulo do $\mathrm{Zn}$ no tecido hepático e na carcaça foi significativamente maior $(P<0,05)$ do que nas brânquias e musculatura (Tabela 2). Estes resultados corroboram com os encontrados na literatura que observaram o fígado como órgão de maior cúmulo de Zn (Murugan et al., 2008; Agah et al. \& Shinn et al., 2009).

Os peixes, devido ao excelente valor nutricional, são amplamente consumidos em várias partes do mundo (Ikem \& Egiebor, 2005). Entretanto, a contaminação difusa das águas pode levar ao acúmulo de metais em tecidos de diferentes espécies de peixes, incluindo os destinados ao consumo humano (Arain et al.,2008; Palaniappan et al., 2010). Obviamente, a concentração de metais nos peixes será maior em locais contaminados, especialmente em áreas de grande atividade industrial, o que demonstra o impacto da atividade humana sobre o meio ambiente (Kosanovic et al., 2007; Mendil et al., 2010b).

Em todas as espécies analisadas, as menores concentrações de $\mathrm{Zn}$ foram observadas na musculatura, significativamente menores $(P<0,05)$ aos valores encontrados nas brânquias, fígado e carcaça (Tabela 2). Na musculatura, o maior valor observado do $\mathrm{Zn}$ foi de $17,92 \mathrm{mg} / \mathrm{kg}$ em uma amostra da espécie Pimelodus sp. (Figura 1).

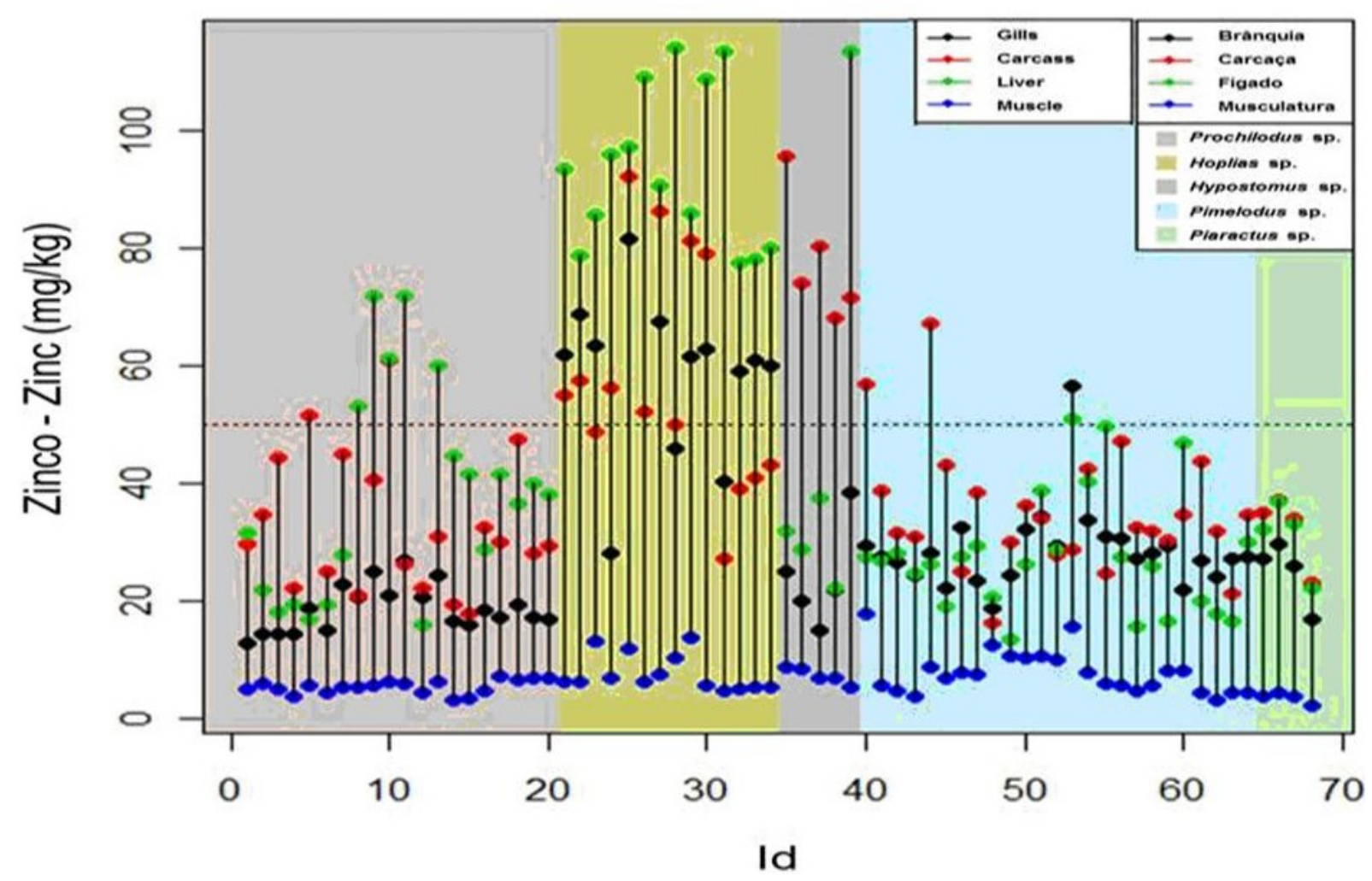

Figura 2 - Concentração de Zn nas diferentes estruturas dos 68 animais capturados (Id). As linhas verticais indicam os animais coletados; e cada ponto sua respectiva estrutura. As cores de fundo representam as diferentes espécies. A linha horizontal tracejada representa a concentração máxima permitida pela legislação brasileira $(50 \mathrm{mg} / \mathrm{Kg})$. 
Tabela 1 - Espécie, peso (g) e comprimento (cm), desvio padrão e valores máximos e mínimos de peixes capturados no Rio São Francisco, 2008

\begin{tabular}{|l|c|c|}
\hline Espécie & Peso $(\mathrm{g})$ & Comprimento (cm) \\
\hline Hoplias sp. & $717,07+/-337,71(320-1410)$ & $36,79+/-5,03(30-45)$ \\
\hline Hypostomus sp. & $121,49+/-81,22(71-264)$ & $19,90+/-3,64(16,51-26,00)$ \\
\hline Piaractus $s p$. & $251,25+/-36,71(212-295)$ & $19,88+/-1,93(18,00-22,50)$ \\
\hline Pimelodus sp. & $112,40+/-54,23(32-275)$ & $20,44+/-3,64(12-31)$ \\
\hline Prochilodus sp. & $534,10+/-390,69(312-2100)$ & $30,63+/-5,02(24,50-48,00)$ \\
\hline
\end{tabular}

Tabela 2 - concentração media e desvio padrão de zinco $(\mathrm{mg} / \mathrm{kg})$ nas vísceras de peixe capturados no Rio São Francisco, 2008.

\begin{tabular}{|c|c|}
\hline Órgão & Zn $(\mathrm{mg} / \mathrm{kg})$ \\
\hline Brânquias & $31,27+/-16,39^{\mathrm{b}}$ \\
\hline Carcaça & $42,20+/-18,94^{\mathrm{C}}$ \\
\hline Fígado & $45,93+/-29,63^{\mathrm{C}}$ \\
\hline Musculatura & $6,82+/-3,00^{\mathrm{a}}$ \\
\hline
\end{tabular}

$C V(\%)=49,97$. Letras distintas diferem entre si estatisticamente pelo teste de Tukey, $P<0,05$

Resultados semelhantes foram observados por Kosanovic et al. (2007), os quais também encontraram valores altos de $\mathrm{Zn}$ no fígado, acima de $50 \mathrm{mg} / \mathrm{kg}$ e na musculatura abaixo de $10 \mathrm{mg} / \mathrm{kg}$.

A musculatura é a parte do peixe de maior valor econômico e a mais importante para a alimentação humana segundo Kosanovic et al. (2007). No presente trabalho, a concentração de $\mathrm{Zn}$ neste tecido é considerada segura para o consumo humano de acordo com os limites estipulados pela Anvisa (1965). Entretanto, a capacidade cumulativa dos metais deve ser considerada como um fator de risco, principalmente para populações que possuem altas taxas de consumo de peixes, como a população ribeirinha. A análise do tecido muscular como bioindicador de contaminação ambiental não é um bom índice para avaliar as reais condições de poluição de um ecossistema, já que este não é um tecido de eleição para o acúmulo de Zn. Fato demonstrado pelos valores sempre baixos comparados aos outros tecidos (Espinoza-Quiñones et al., 2010; Palaniappan et al., 2010). Contudo, a concentração do $\mathrm{Zn}$ no tecido hepático seria um excelente indicador de poluição, já que este órgão apresentou os maiores acúmulos deste elemento (Tepe et al., 2008; Agah et al., 2009).

Foi avaliada, neste estudo, a influência do comprimento e do peso dos animais sobre a concentração de $\mathrm{Zn}$. Foram encontradas correlações de 0,28 e 0,21, respectivamente, que apesar de baixos, são positivos, corroborando com a literatura. Poderia ser esperada uma maior concentração de metais em peixes, com o tamanho crescente desses animais. Não obstante, o crescimento deve ser lento em relação à taxa de acumulação dos elementos (Leung et al., 2001).
$O$ peso e comprimento médios das cinco espécies de peixes analisadas, assim como o desvio padrão e os valores máximos e mínimos, estão demonstrados na tabela 1. De maneira contrária, muitos trabalhos relatam correlações negativas entre a concentração de $\mathrm{Zn}$ e o comprimento e peso dos animais, ou seja, as maiores concentrações de Zn foram observadas em animais menores, logo, nos espécimes mais jovens. Este fato é justificado pela maior atividade metabólica e, portanto, maior acúmulo de $\mathrm{Zn}$ nos juvenis e, também, pelo crescimento mais rápido em relação à acumulação (Canli \& Atli, 2003; Agah et al., 2009). Além do tamanho e peso dos peixes, a influência do hábito alimentar e comportamento dos animais sobre a acumulação de $\mathrm{Zn}$ foram avaliados. A espécie Piaractus sp. foi a única, dentre as estudadas, que não apresentou nenhuma amostra analisada com valores acima de $50 \mathrm{mg} / \mathrm{kg}$ (Figura 2). A espécie Hoplias sp. apresentou as maiores concentrações médias de $Z n(P<0,05)$ nas brânquias $(58,18 \mathrm{mg} / \mathrm{kg})$ e fígado $(93,50 \mathrm{mg} / \mathrm{kg})$ quando comparado a outras espécies (Figura 2). Já na carcaça, o maior valor foi encontrado na espécie Hypostomus sp. $(77,93 \mathrm{mg} / \mathrm{kg})$, seguido da espécie Hoplias sp. $(57,93 \mathrm{mg} / \mathrm{kg})$. Todos os valores citados ultrapassam o estipulado pela ANVISA (1965; Figura 2). Diferentes concentrações de metais entre espécies distintas são esperadas já que estes elementos podem ser bioacumulados ao longo da cadeia trófica (Canli \& Atli 2003; Chiu \& Mok 2010). Desta forma, é esperado maior acúmulo de $\mathrm{Zn}$ em espécies que ocupam o topo da cadeia alimentar. Esse padrão de acúmulo de metais foi observado neste trabalho. A espécie Hoplias sp. ocupa o topo da cadeia alimentar aquática e, portanto, alimenta-se basicamente de outros peixes (Oyakawa \& Mattox, 2009). Neste 
estudo, esta espécie apresentou altos valores médios de Zn, de forma que as concentrações médias nas brânquias, carcaça e fígado ultrapassaram o estipulado por lei. Resultados semelhantes foram observados em estudo conduzido por Gobbi et al. (2010) no Rio São Francisco (MG) com a espécie Pseudoplatystoma coruscans (Surubim), peixe carnívoro de alto valor comercial. Neste trabalho, $30 \%$ do total de amostras analisadas estavam fora do padrão legal. O fígado foi o órgão de maior acúmulo de $\mathrm{Zn}, 95 \%$ das amostras deste órgão estavam irregulares de acordo com a ANVISA (1965). Uma parte significativa dos metais introduzidos no rio pode acumular-se nos sedimentos através de processos químicos e físicos (Silva et al., 2009). E, portanto, os sedimentos representam um importante local de depósito de metais no ambiente aquático que estão, muitas vezes, em concentração maior do que a encontrada na água, representando, desta forma, um risco direto para a alimentação de organismos bentônicos. Além disto, também pode representar uma importante fonte de contaminação, em longo prazo, para toda a cadeia trófica local (Mendil et al., 2010b). Saei-Dehkordi \& Fallah (2011) observaram que animais demersais apresentaram concentrações superiores aos que nadam livremente na coluna de água. Da mesma forma, neste trabalho, a espécie Hypostomus sp., que se alimenta junto ao fundo do rio, em contato com os sedimentos, apresentou altas concentrações de Zn. Estes peixes possuem comportamento bentônico e são considerados consumidores primários. Deste modo, podem ser observados explorando toda a cobertura vegetal e colônias de algas do fundo do rio (Casatti \& Castro, 1998; Garavello \& Garavello, 2004). Diante dos hábitos alimentares e comportamentais de Hypostomus sp. e Hoplias sp., e da característica bioacumulativa dos metais, sugere-se a utilização destas espécies como biomarcadores de contaminação ambiental.

Este estudo também avaliou a concentração de Zn nos diferentes pontos de coletas. Não foi observada diferença $(P>0,05)$ da concentração deste metal nos peixes entre os cinco pontos estudados do Rio São Francisco (Figura 1), possivelmente devido aos hábitos migratórios dos peixes. Além disto, a contaminação pode estar ocorrendo ao longo do leito do rio decorrente de diferentes fontes poluidoras que poderiam prejudicar uma extensa área. Pesquisa realizada por Gomes et al., (2010) na mesma região do Rio São Francisco deste estudo, demonstrou altos valores de $\mathrm{Zn}$ no sedimento superficial do rio na região do ponto 3 (altitude: $511,28 m-18^{\circ} 111^{\prime} 53^{\prime \prime} \mathrm{S}-45^{\circ} 15^{\prime} 06^{\prime \prime} \mathrm{W}$, declinando até a jusante do rio Abaeté, ponto 5 (altitude: $\left.506,10 \mathrm{~m}-18^{\circ} 02^{\prime} 26^{\prime \prime} \mathrm{S}-45^{\circ} 11^{\prime} 15^{\prime \prime} \mathrm{W}\right)$, com concentração do metal semelhante à encontrada no reservatório da Usina Hidrelétrica de Três Marias, ponto 1 (altitude: $548,78 \mathrm{~m}$ - $18^{\circ} 12^{\prime} 12^{\prime \prime} \mathrm{S}$ $\left.45^{\circ} 19^{\prime} 28^{\prime \prime} \mathrm{W}\right)$. Esses resultados sugerem que a região estudada está ameaçada pela poluição oferecendo risco à toda biota do ecossistema em questão.

\section{CONCLUSÕES}

Níveis acima de $50 \mathrm{mg} / \mathrm{kg}$ de $\mathrm{Zn}$ foram observados em 28 dos 68 peixes utilizados neste estudo. Das 272 amostras estudadas, 50 (brânquias, fígado e carcaça) estavam impróprias para o consumo, sendo que a musculatura dos peixes apresentou baixas concentrações deste metal. Nas espécies que Hoplias sp. e Hypostomus sp. foram encontradas as maiores concentrações de Zn, especialmente no fígado, podendo ser estas utilizadas como bioindicadores de poluição. Não foram observadas diferenças da concentração de $\mathrm{Zn}$ entre os diferentes pontos de coleta, sugerindo que a contaminação do rio é difusa.

\section{AGRADECIMENTOS}

Agradecimento Especial à Fundação de Amparo à Pesquisa do Estado de Minas Gerais (FAPEMIG), ao Conselho Nacional de Desenvolvimento Científico e Tecnológico (CNPq) e à Coordenação de Aperfeiçoamento de Pessoal e Nível Superior (CAPES).

Contribuição apresentada no "International Symposium Workshop on Mining activities, refineries, pollution control and remediation strategies", realizado no IGC-UFMG (Belo Horizonte, 12 a 20 de setembro de 2011).

\section{REFERÊNCIAS BIBLIOGRÁFICAS}

Agah H., Leermakers M., Elskens, M., Fatemi S.M., Baeyens, W. 2009. Accumulation of trace metals in the muscle and liver tissues of five fish species from the Persian Gulf. Environ. Monit. Assess., 157, 499-514.

American Public Health Association (APHA). 1992. Standard methods for examination of water and wastewater.. New York, v.16, $1219 \mathrm{p}$

Amundesen P.A., Staldvik F.J., Lukin A.A., Kashulin N.A. Papova O.A., Reshetnilkov Y.S. 1997. Heavy metal contamination in freshwater fish from the borderregion between Norway and Russia. Sci. Total Environ., 201, 21-224.

ANVISA 1965. Limite máximo de tolerância de contaminantes inorgânicos em alimentos. Decreto $\mathrm{N}^{\circ}$ 55.871, de 26 de Março de 1965, Brasil.

Arain M.B., Kazi T.G., Jamali M.K., Jalbani N., Afrid H.I., Shah A. 2008. Total dissolved and bioavailable metals in water and sediment samples and their accumulation in Oreochromis mossambicus of polluted Manchar Lake. Chemosphere, v. 70, p1845-1856.

Ashraf W., Seddigi, Z., Abulkibash, A., Khalid M. 2006. Levels of selected metals in canned fish consumed in Kingdom of Saudi Arabia. Environ. Monit. Assess. v. 117, p271-279. 
Canli M., Atli G. 2003. The relationships between heavy metal ( $C d$, $\mathrm{Cr}, \mathrm{Cu}, \mathrm{Fe}, \mathrm{Pb}, \mathrm{Zn}$ ) levels and the size of six Mediterranean fish species. Environ. Pollut., v.121, p129-136.

Casatti L., Castro R.M. C. 1998. A fish community of the São Francisco River headwatersriffles, southeastern Brazil. Ichthyol. Explor. Freshwaters, v.9, p.229-242.

Chiu K. H., Mok H.K. 1996. Study on the accumulation of heavy metals in Shallow-Water and Deep-Sea Hagfishes. Arch. Environ. Contam. Toxicol., 60, p643-653.

Espinoza-Quiñones F.R., Módene A.N., Palácio S. M., Szymanski N., Welter R.A. Rizzutto M.A., Borba C.E., Kroumov A.D. 2010. Evaluation of trace element levels in muscles, liver and gonad of fish species from São Francisco River of the Paraná Brazilian state by using SR-TXRF technique. Appl. Radiat. Isot., 68, p2202-2207.

Garavello J.C., Garavello J.P. 2004. Spatial distribution and interaction of four species of the catfish genus Hypostomus lacépède with bottom of rio São Francisco, Canindé do São Francisco, Sergipe, Brazil (Pisces, Loricariidae, Hypostominae). Braz. J. Biol., 64, p591-598.

Gobbi J.M. 1998. Estudo sobre a presença de metais em diferentes tecidos de peixes surubins (Pseudoplatystoma coruscans) capturados no Rio São Francisco (MG). Dissertação de Mestrado, Escola de Veterinária, Universidade Federal de Minas Gerais - UFMG, 76p.

Godinho H.P., Godinho A. L. 2003. Águas, peixes e pescadores do São Francisco das Minas Gerais. Belo Horizonte: PUC Minas $468 p$.

Gomes M.V.T., Costa, A.S., Garcia C.A.B., Passos E.A., Alves J.P. 2010. Concentrações e associações geoquímicas de $\mathrm{Pb}$ e $\mathrm{Zn}$ em sedimentos do rio São Francisco impactados por rejeitos da produção industrial de zinco. Química Nova, 33, p20882092.

Hogstrand C., Wilson R.W., Polgar, D., Wood C. M. 1994. Effects of zinc on the kinetics of branchial calcium uptake in freshwater rainbow trout during adaptation to waterborne zinc. J. Exp.. Biol., 186, p55-73.

Ikem, A.; Egiebor, N.O. 2005. Assessment of trace elements in canned fish (mackerel, tuna, salmon, sardines and herrings) marketed in Georgia and Alabama (United States of America). J. Food Compost. Anal., 18, p771-787.

Kosanovic M., Hasan M.Y., Subramanian D., Al Ahbabi A.A., Al Kathiri O.A., Aleassa, E.M., Adem A. 2007. Influence of urbanization of the western coast of the United Arab Emirates on trace metal content in muscle and liver of wild Red-spot emperor (Lethrinus lentjan). Food and Chem. Toxicol., 45, p2261-2266.

Kuz'mina V.V. 2011. The influence of zinc and copper on the latency period for feeding and the food uptake in common carp, Cyprinus carpio L. Aquatic Toxicol., 102, p73-78.

Leung K.M.Y., Morgan I.J., Wu R.S.S., Lau T.C., Svavarsson J., Furness R.W. 2010. Growth rate as a factor confounding the use of the dogwhelk Nucella lapillus as biomonitor of heavy metal contamination. Mar. Ecol. Progress Series, 221, p145159.

Lins J.A.P.N., Kirschnik P.G., Queiroz V.S., Cirio S.M. Ed.8. 2010. Uso de peixes como biomarcadores para monitoramento ambiental aquático. Revista Acadêmica: Ciências Agrárias e Ambientais, p469-484.

Mansilla-Rivera, I.; Rodríguez-Sierra, C.J. 2011. Metal levels in fish captured in Puerto Rico and estimation of risk from fish consumption. Arch. Environ. Contamin. Toxicol., 60, p132144.
Marques D.C., Matta S.L.P., Oliveira J.A., Dergam J.A. 2009. Alterações histológicas em brânquias de Astyanax aff. bimaculatus causadas pela exposição aguda ao zinco. In: XVI Congresso Brasileiro de Toxicologia, Belo Horizonte, 2009. Rev. Bras. Toxicol., 22, 26.

Mendil D., Demirci Z., Tuzen M., Soylak M. 2010a. Seasona investigation of trace element contents in commercially valuable fish species from the Black sea, Turkey. Food Chem. Toxicol., 48, p865-870.

Mendil D., Ünal Ö.F., Tüzen M., Soylak M. 2010b. Determination of trace metals in different fish species and sediments from the River Yes ilırmak in Tokat, Turkey. Food Chem. Toxicol., 48, p1383-1392.

Mottin E., Caplat C., Mahaut M.L., Costil K., Barillier D., Lebel J.M., Serpentini A. 2010. Effect of in vitro exposure to zinc on immunological parameters of haemocytes from the marine gastropod Haliotis tuberculata. Fish Shellfish Immunol., 29, p846-853.

Murugan S.S., Karuppasamy R., Poongodi K., Puvaneswari S. 2008 Bioaccumulation pattern of zinc in freshwater fish Channa punctatus (Bloch.) after chronic exposure. Turk. J. Fish. Aquatic Sci., 8, p55-59.

Nakayama S.M.M., Ikenaka Y., Muzandu K., Choongo, K., Oroszlany B., Teraoka H., Mizuno N., Ishizuka M. 2010. Heavy metal accumulation in lake sediments, fish (Oreochromis niloticus and Serranochromis thumbergi), and crayfish (Cherax quadricarinatus) in Lake Itezhi-tezhi and Lake Kariba, Zambia. Arch. Environ. Contamin. Toxicol., 59, p291-300.

Oyakawa O.T., Mattox G.M. 2009. Revision of the neotropical trahiras of the Hoplias lacerdae species-group (Ostariophysi: Characiformes: Erythrinidae) with descriptions of two new species. Neotrop. Ichthyol., 7, p117-140.

Ozden O. 2010. Micro, macro mineral and proximate composition of Atlantic bonito and horse mackerel: a monthly differentiation. Internat. J. Food Sci. Technol., 45, p578-586.

Palaniappan L.R.M., Nishanth T., Renju V. B. 2010 Bioconcentration of zinc and its effect on the biochemical constituents of the gill tissues of Labeo rohita: An FT-IR study. Infrar. Physics Technol., 53, p103-111.

Saei-Dehkordi S.S., Fallah A.A. 2011. Determination of copper, lead, cadmium and zinc content in commercially valuable fish species from the Persian Gulf using derivative potentiometric stripping analysis. Microchem. J., in press.

Sampaio I.B.M. 2007 Estatística aplicada à experimentação animal. 3 ed. Belo Horizonte: Fundação de Ensino e Pesquisa em Medicina Veterinária e Zootecnia 264p.

Shinn C., Dauba F., Grenouillet G., Guenard G., Lek S. 2009. Temporal variation of heavy metal contamination in fish of the river lot in southern France. Ecotoxico. Environ. Safety, 72, p1957-1965.

Silva E.F, Almeida S.F.P., Nunes M.L., Luís A.T., Borg F., Hedlund M., Sá C.M., Patinha C., Teixeira P. 2009. Heavy metal pollution downstream the abandoned Coval da Mó mine (Portugal) and associated effects on epilithic diatom communities. Sci. Total Environ. 407, p5620-5636.

Sloman K.A. 2007. Effect of trace metals on salmonid fish. The role of social hierarchies. Applied Animal Behaviour Science, 104, p326-345.

Szyczewski P., Siepak J., Niedzielski P. 2009. Research on heavy metals in Poland. Polish J. Environ. Studies, 18, p755-768.

Tepe Y., Türkmen M., Türkmen A. 2008. Assessment of heavy metals in two commercial fish species of four Turkish seas. Environ. Monit. Assess., 146, p277-284. 